INVESTIGACIÓN/RESEARCH

\title{
LA GESTIÓN DE COMPETENCIAS INFORMACIONALES EN LAS UNIVERSIDADES: RETO PARA LOS PROFESIONALES DE LA INFORMACIÓN
}

Marlery Sánchez Díaz: Universidad de La Habana. Cuba. infctf@biocen.cu

\section{RESUMEN}

El tema de las competencias se irradia en la docencia universitaria a partir de que es imprescindible lograr en los estudiantes los conocimientos, habilidades y actitudes pertinentes a lo que necesita la sociedad. Dentro de éstas se encuentran las competencias informacionales y su gestión es un reto para los profesionales de la información. En este trabajo se demuestra tal presupuesto a partir de la literatura publicada y de la investigación de campo realizada por la autora en su tesis doctoral. Para ello se definen las competencias informacionales y en qué consiste su gestión a partir de la explicación de cada una de sus etapas. Y se desarrolla la gestión de las competencias informacionales teniendo en cuenta diferentes ámbitos: los profesionales de la información, los docentes y los estudiantes.

PALABRAS CLAVE: Alfabetización - Competencias - Información - Universidad Docencia

\footnotetext{
${ }^{1}$ Autor correspondiente: Marlery Sánchez Díaz: Profesora Asistente Adjunta del Departamento de Ciencias de la Información de la Facultad de Comunicación en la Universidad de La Habana. Cuba.

Correo: infctf@biocen.cu
} 


\title{
INFORMATION SKILLS MANAGEMENT IN UNIVERSITIES: CHALLENGE FOR INFORMATION PROFESSIONALS
}

\begin{abstract}
The topic of competencies in university teaching radiates from it is essential to bring in students the knowledge, skills and attitudes relevant to what society needs. Among these are information skills and their management is a challenge for information professionals. This paper demonstrates that budget from the published literature and field research conducted by the author in his doctoral thesis. This information skills are defined and what its management from the explanation of each of its stages. And develops management information skills considering different areas: information professionals, teachers and students.
\end{abstract}

KEY WORDS: Literacy - Competencies - Information - University - Teaching

\section{INTRODUCCIÓN}

El tema de las competencias, se irradia en la docencia universitaria; así se plantea "Los titulados que salen de la universidad deben ser competentes; por ende la organización de la universidad, la oferta de las Licenciaturas y la calidad de los servicios deben orientarse hacia el aprendizaje por competencias." (Martínez, 2005) "El ambiente y la filosofía de los procesos educacionales está cambiando, particularmente en el área de la educación superior, donde el aprendizaje basado en las competencias y habilidades está haciéndose cada vez más." (Pinto, y otros, 2008a)

Partiendo de este enfoque, y tomando en cuenta lo que expresan los diferentes autores "La información es un recurso esencial de la 'nueva economía' y el desarrollo de las tecnologías de información y comunicación están estrechamente vinculadas a los papeles protagonistas de este nuevo entorno (...)" (Pinto, y otros, 2009b); "Cada uno de los miembros de la sociedad necesita (...), por sus propios medios, la información que le requiere su trabajo, su estudio y hasta su vida cotidiana." (Angulo, 2003) y que el concepto de formación a lo largo de la vida imperante supone la adquisición y actualización continua para el desarrollo correcto tanto en el estudio, en la profesión como en lo cultural; la gestión de competencias informacionales se hace imprescindible en cualquier contexto. Así se plantea "La universidad debe responder a nuevas expectativas como el aprendizaje a lo largo de la vida, el desarrollo de habilidades multidisciplinares y la alfabetización informacional (...)" (Pinto, y otros, 2008b)

El sistema educativo debe preparar a los estudiantes hacer frente a la realidad, es por eso que el nuevo Espacio Europeo de Educación Superior; se basa, entre otros conceptos, en el de competencias, destacando la relevancia de las informativas y enfatiza en el rol de las universidades en la Europa del conocimiento. Es decir "La alfabetización informacional-digital se ha convertido en una necesidad y en una 
exigencia de los actuales sistemas de enseñanza universitaria en el marco de la Europa del Conocimiento." (Pinto, y otros, 2009a)

Las competencias informacionales, están siendo objeto de atención dentro de la Bibliotecología y la Ciencia de la Información. "Así, no es de extrañar que la alfabetización informacional, como adquisición de competencias informacionales para desarrollar la propia educación a lo largo de la vida (...), sea un tema que suscite cada vez más la atención de los estudios de Biblioteconomía y Documentación." (Pinto, y otros, 2009a)

Se entiende por profesional de la información "aquel que tiene un saber especializado en relación con la información y este saber lo pone al servicio de otros, dicho saber se utiliza para mejorar las tareas intelectuales de las personas, y realizar su trabajo con rapidez y eficacia." (Sánchez, 2004); pero su actuación profesional ha cambiado en el cursar del tiempo.

En la civilización antigua tenía como función principal la salvaguarda, organización, conservación y custodia de los depósitos documentales de los palacios y templos. En el Antiguo Oriente, fueron los sacerdotes los que se iniciaron en esta ocupación, y su labor consistía en reunir, transcribir, organizar y conservar los documentos. En Occidente, es en Alejandría, donde con más claridad surge la figura del bibliotecario (...) En la Roma antigua, los primeros trabajadores de la información fueron, en unos casos, esclavos y, en otros, funcionarios públicos oficialmente reconocidos; que dentro de sus funciones esenciales estaba la de organización y cuidado de los fondos. En la Edad Media, en las catedrales y monasterios, los monjes copiaban, ilustraban y restringían el uso de los volúmenes que guardaban. Con el Renacimiento, los trabajadores de la información tuvieron que enfrentarse a una demanda diferente de libros. (Sánchez, 2004).

Durante el siglo XIX, el movimiento de bibliotecas públicas introdujo una nueva concepción en esta institución que exigió un trabajador de la información renovado. (Frías, 2000)

Los cambios en el siglo XX, sobre todo en la esfera tecnológica, produjeron una verdadera revolución en las concepciones sobre su rol profesional. En la nueva sociedad, dicho profesional ha rebasado sus recintos tradicionales -bibliotecas, archivos, centros de documentación, centros de información- y su labor alcanza cualquier instancia donde se genere, cree, manipule y se acceda a la información. Y ya a mediados de los años 80, a partir de la definición de information literacy dada por la ALA, "algunos bibliotecarios norteamericanos empiezan a construir un escenario en el que ellos se atribuyen un papel central (...)" (García, y otros, 2008)

Y según Dudziak (Dudziak, 2003) es en los años 90 que se empiezan a implementar programas de information literacy en diversos lugares del mundo; siendo el principal propósito de bibliotecarios especialmente en la enseñanza universitaria. 
En este siglo XXI, en el discurso sobre competencias informacionales cada vez más se asume al bibliotecario como educador; presente de manera creciente desde los últimos años en diversos foros, eventos y revistas.

En Scopus aparecen 693 registros que responden a la relación de la gestión de competencias informacionales con el profesional de la información; predominando los autores cuya afiliación responde a universidades y los años 2011 y 2010 son los más prolíferos con 107 y 105 contribuciones respectivamente.

Como plantea Gómez-Hernández “El servicio de alfabetización informacional (Alfin), $\mathrm{y}$ en general el ejercicio de una función educativa y de apoyo al aprendizaje, va extendiéndose en la práctica profesional y en las bibliotecas de un modo progresivo pero lento, y no exento de dificultades y altibajos." (Gómez-Hernández, 2008)

De lo planteado se desprende el siguiente objetivo general: demostrar que la gestión de las competencias informacionales en la universidad es un reto para los profesionales de la información en ejercicio. Este trabajo se desarrolla a partir de la revisión de la literatura y de la investigación de campo realizada para la tesis doctoral de la autora.

\section{METODOLOGÍA}

La metodología empleada se basa en el análisis de fuentes primarias para aplicar el método inductivo-deductivo, por el que, a partir de determinados principios, teorías o leyes se derivan supuestos a mediante los que se explicarán los casos particulares de la Alfabetización en competencias informacionales.

A partir de ahí recurriremos al método de modelación. Esta consiste en la representación teórica de los objetos, o fenómenos, o particularidades de los elementos que confluyen en el proceso de alfabetización, lo que permite descomponerlos, abstraer determinadas cualidades, operar y experimentar con él.

Todo esto nos permitirá formular y reformular definiciones de conceptos ya que es importante en el desarrollo de toda investigación -y la presente se incardina dentro de la línea de trabajos de la autora-, precisar en que sentido se emplean términos que pueden tener diferentes acepciones vinculándolo con las variables e indicadores a través de los cuales se expresa su significado dentro de la investigación. Así se sientan las bases de una construcción teórica (basada en el empirismo de la analítica documental) que puede ser reutilizada y reformulada según las adaptaciones precisas en cada pesquisa.

\section{ANÁLISIS Y DISCUSIÓN}

Las competencias informacionales, consisten en poner en acción, en práctica, en juego, en movilización; de forma combinada, mezclada o integrada; en un contexto y con un contenido determinado (transferibles), todos los recursos (habilidades, conocimientos, 
actitudes); para solucionar con éxito problemas y aprender a aprender, a partir de la interacción efectiva con la información; sin delimitación de tipo, formato y soporte. Son necesarias en cualquier ámbito e incluyen otras competencias como las tecnológicas, las bibliotecarias y el pensamiento crítico. (Sánchez, 2008)

Es preciso administrar estas competencias con el fin de obtenerlas en grado óptimo y a un nivel de excelencia. Para ello es necesario atravesar por diferentes etapas o momentos (Esquema 1): identificación, normalización, evaluación, formación y certificación. (Sánchez, 2010)

\section{Esquema 1. Etapas de la gestión de C.I.}

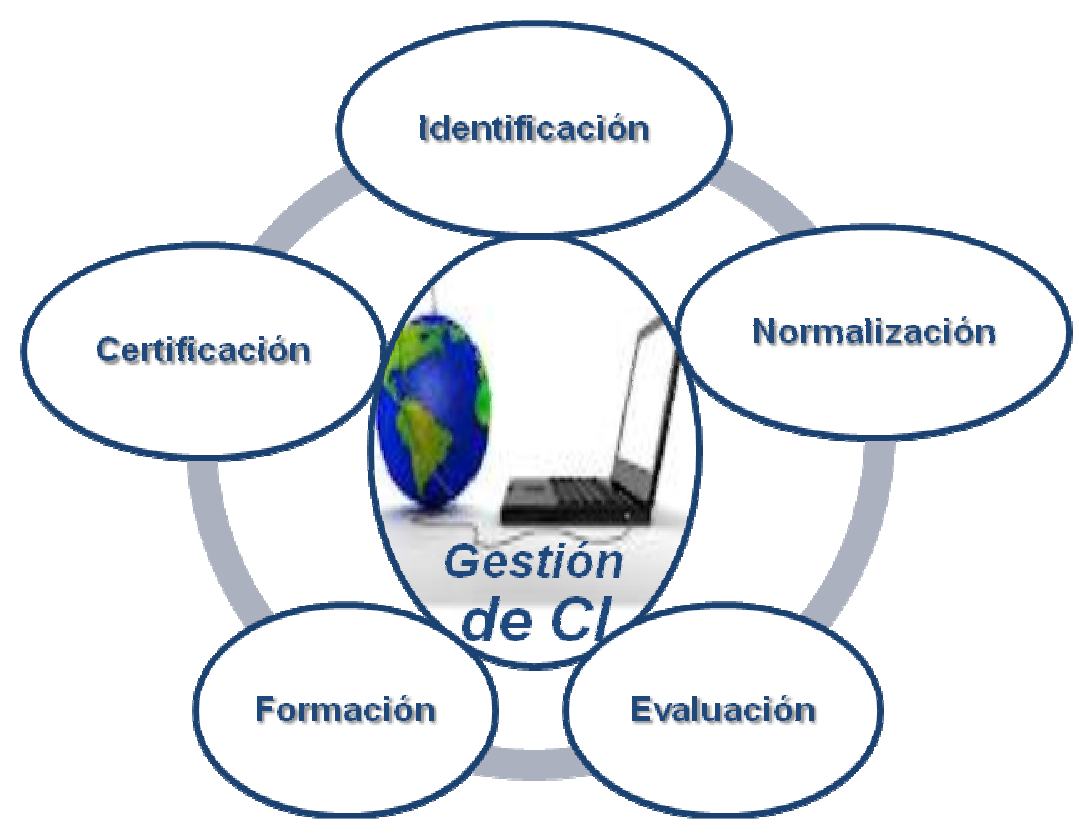

Tomando en cuenta el enfoque de competencias laborales (Gallart, y otros, 1995) (Mirabile, 1997) (Irigoin, 1998) (Cardona, y otros, 1999) (Vargas, 2004) se puede plantear que la identificación de competencias informacionales consiste en el establecimiento de las competencias relacionadas con la información, que determinan el desempeño exitoso de los individuos en una actividad determinada. Esta se ha realizado a través del desarrollo de modelos, de acuerdo con las particularidades y expectativas de escenario y circunstancia. En el marco del proyecto canadiense Test d'identification de competentes infomationnelles (mapaweb.umontreal.ca/bernh/TICI) se identifican más de 60 modelos a escala internacional. Dentro de los que se encuentran: Modelo de Marland (Marland, 1981); Recalling, Explaining, Analyzing, Challenging, Transforming and Synthesizing (REACTS) (Stripling, y otros, 1988); Information Seeking Process (ISP) (Kuhlthau, 1989); Big Six Skills Information Problem-Solving (Eisenberg, y otros, 1990); Modelo de Doyle (Doyle, 1992); Pathways of knowledge, Pappas y Tepe (Pappas, y otros, 1995); Purpose, Location, Use and Self-evaluation (PLUS) (Herring, 1996); 'Las siete caras de la alfabetización informacional' (Bruce, 1997); HEBORI (Benito, 2000); The Learning Connection Information Literacy and the Students (Lupton, 
2004); Panning for Gold: Influencing the Experience of Web-based Information Searching (Edwards, 2006); Modelo de Markless (Markless, 2008).

A partir de los diferentes autores (Ducci, 1997) (Laur-Ernst, 2000) (Tucker, y otros, 2000) (Vargas, y otros, 2001) (Catalana, y otros, 2004) hay que decir que la normalización de competencias informacionales es el proceso de institucionalización de un estándar de competencias relacionadas con la información, donde se convierte a cada competencia en una norma acordada. La mayoría de las normas de competencias informacionales han surgido de las diferentes asociaciones: ACRL2 (ACRL, 2000), (CAUL, 2001) Estadounidenses; ANZIIL $^{3}$ (ANZIIL, 2004), Australiana; SCONUL4 (SCONUL, 1999) y CILIP5, Británicas; Ministere de l'Education Nationale, de la Recherched et de la Technologie, Francesa; IFLA, Internacional.

Siguiendo la literatura (Hager, y otros, 1994) (McDonald, y otros, 1995) (Cardona, y otros, 1999) (Jornet, y otros, 2002) (Catalana, y otros, 2004) la evaluación de competencias informacionales es el proceso de verificación de evidencias acerca del desempeño relacionado con la información, de un individuo contra el estándar definido en la norma. Ahora bien los tipos de evaluaciones más frecuentes en competencias son: "la diagnóstica, la de los procesos de aprendizaje y la de resultados" (Catalana, y otros, 2004). Esa es la razón por la cual se habla de evaluación antes, durante y/o después de la etapa de formación de competencias. Se han desarrollado diferentes iniciativas al respecto: SAILS Standardized Assessment of Information Literacy Skills, - ETS/ICT- Educational Testing Service/Information and Communications Technology www.ets.org/ictliteracy/, Bay Area Community College Information Competency Assessment Project', ISST Information-Seeking Skills Test (www.lib.jmu.edu/gold/isst.htm), TRAILS-Tool for Real-time Assessment of Information Literacy Skills ${ }^{8}$. Proyecto del Institute for Library and Information Literacy Education en Estados Unidos, Information Skills Survey (ISS) for Assessment of Information Literacy in Higher Education, European Network on Information Literacy for a Culture of Information ENIL.

Al enmarcar la etapa de formación de competencias laborales (Ameida de, 1991) (Beedle, 1997) (Mertens, 1998) (Ibarra, 2000) (Vargas, y otros, 2001) (Catalana, y otros, 2004) en la perspectiva informacional; se puede decir que consiste en el proceso de enseñanza-aprendizaje que facilita la adquisición de las competencias informacionales al nivel requerido. Tomando en cuenta la literatura (Tejada, 1999) (Pasadas, y otros, 2007) (Badke, 2008) en el Esquema 2 se muestran las formas de adquirirlas.

\footnotetext{
2 Association of College and Research Libraries

${ }^{3}$ Instituto Australiano y Neozelandés para la Alfabetización Informacional

4 Society of College, National and University Libraries

${ }^{5}$ Chartered Institute of Library and information Professionals.

${ }^{6}$ www.projectsails.org

7 (http:/ / www.topsy.org/ICAP)

8 (www.trails-9.org/).
} 


\section{Esquema 2. Forma de adquirir las competencias}

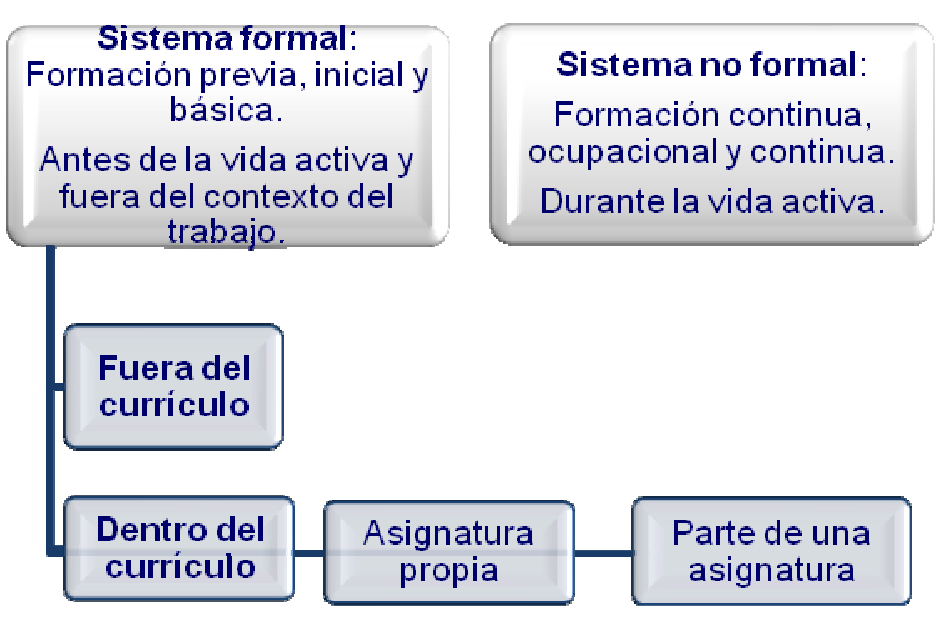

Mediante el enfoque de competencias laborales (INEM, 1995) (Agudelo, 1997) (Irigoin, y otros, 2002) se puede decir que la certificación de competencias informacionales es el reconocimiento oficial que avala las competencias relacionadas con la información demostradas por los individuos acorde a lo establecido independientemente de cómo fueron adquiridas.

Como se plantea en la literatura "La introducción de competencias informacionales en el marco de la enseñanza superior debe tener en cuenta todos los contextos en los que se precisa poner en marcha dichas competencias." (Roca, 2006) Así en este entorno las etapas de la gestión de competencias informacionales deben abarcar a los profesionales de la información, a los docentes y a los estudiantes.

En cuanto a los profesionales de la información, lo primero, es que es imprescindible dominen conceptos, modelos, normas y experiencias en competencias informacionales; porque en muchos casos no comprenden la dimensión de la alfabetización informacional más allá de la formación de usuarios. "(...) deben conocer cómo se entienden en la actualidad los servicios Alfin, y cómo se pueden programar para que logren contribuir a la competencia informacional de sus usuarios." Es por ello por lo que deben involucrarse en el tema, revisar literatura relevante y actualizada sobre Alfin y participar en eventos y cursos relacionados con el tema.

Por otra parte, para gestionar las competencias informacionales, los profesionales de la información deben ser competentes informacionalmente en un contexto determinado y con un contenido específico. Así en la literatura se plantea “(...) los bibliotecarios deben tener su propia alfabetización o 'competencia informacional', es decir, dominar los conceptos propios del mundo de la información, sus procedimientos y valores para realizar de modo contextualizado, reflexivo e intencional la selección, evaluación, integración, uso y comunicación ética de la información." (GómezHernández, 2008) "El hecho de que sea la persona que interviene en todo el proceso de selección, tratamiento y difusión de la información de un determinado ámbito temático, le 
convierte en la persona más idónea y más capaz para elaborar e impartir sesiones de formación de contenido temático". (Espinós, y otros)

Pero, no en todos los casos son competentes en información, de acuerdo al concepto manejado en este trabajo; por ende se sugiere una autoformación constante y continuada, asistir a clases como oyente, dialogar con los docentes y estudiantes, revisar autores prolíferos y artículos relevantes sobre las temáticas de las titulaciones afines, entre otras.

Ahora bien, ¿están preparados los profesionales de la información para participar como formadores de competencias informacionales? Es cierto, que los bibliotecarios llevan años realizando formación de usuarios y actividades de aprendizaje en el uso de la información; sin embargo en muchos casos "prima su conocimiento de la materia a impartir antes que su capacidad didáctica o de comunicación." (GómezHernández, 2008) Por ello se hace necesario incluir en sus planes de formación el recibir cursos relacionados con pedagogía, didáctica y desarrollo de contenidos.

Para gestionar las competencias informacionales en la universidad los profesionales de la información deben determinar si en el currículo de las titulaciones se incluyen las competencias informacionales, de qué manera, en qué tipo de asignatura, cómo se forman; para ello deben analizar los planes de estudio, hacer entrevistas, asistir a clases como oyente, dialogar con docentes y estudiantes. También deben dominar las líneas de investigación de la facultad y participar en los consejos científicos.

En cuanto a los docentes, lo primero que deben lograr los profesionales de la información es la implicación en el tema del equipo directivo. El lograr en vicedecanos y jefes de carreras la identificación con el tema, sienta las bases para la gestión de las competencias informacionales en la universidad.

Es preciso, a su vez, que todos los docentes dominen qué se entiende por las competencias transversales relacionadas con la información y las cuestiones que forman parte de ella. En muchos casos no todos comprenden a cabalidad la necesidad de la gestión de estas competencias, están sensibilizados con la incorporación en sus clases de contenidos sobre estas competencias, conocen cómo se puede llevar a cabo esta formación, concientizan la necesidad de la cooperación que debe existir entre los profesores y bibliotecarios. "Si bien muchos docentes estarían interesados en dicha colaboración, suelen desconocer la existencia de esta posibilidad, (...), siguen viendo al bibliotecario como gestor de la colección y dispensador de servicios únicamente." (Hartzell, 1997)

En este sentido se recomienda realizar encuentros sobre el tema de las competencias informacionales y enviar correos electrónicos con experiencias concretas. Además los profesionales de la información deben colaborar puntualmente en asignaturas para demostrar su utilidad y llevar a cabo talleres donde los profesores asistan como alumnos con vistas a su formación en CI pues como exponen diferentes autores "Los docentes no ven la figura del bibliotecario con una experiencia específica suficiente en 
su disciplina temática para discernir los materiales adecuados a la asignatura que imparten o trabajo de investigación. (...) Por otra parte, los bibliotecarios comprueban como, a menudo, el profesorado posee escasas u obsoletas competencias en el uso y acceso a la información." (Christiansen, y otros, 2004)

El realizar entrevistas donde los docentes manifiesten su criterio en cuanto a si consideran preciso implementar acciones para la formación de las competencias informacionales en sus asignaturas y cómo llevar a cabo esta formación enriquece el trabajo.

En cuanto a los estudiantes, creemos que su motivación para enfrentar el tema de las competencias es una garantía de éxito, por eso es preciso incluir materiales promocionales en la Web, ubicar propagandas en los murales, realizar charlas dinámicas e informales donde conozcan los beneficios del tema de las competencias informacionales.

Es preciso realizar la identificación y normalización de competencias informacionales necesarias y una evaluación diagnóstica de las mismas que permita identificar las brechas. A partir de todo ello llevar a cabo pruebas pilotos. Las mismas pueden realizarse dentro de asignaturas, donde los profesores coordinadores deben incorporar esta formación dentro del programa, revisar las unidades temáticas, así como los recursos de información seleccionados por el profesional de la información. Se deben introducir los nuevos contenidos por medio de la exposición, donde se explique, demuestre, ilustre y se manejen situaciones problemáticas simuladas de manera creativa. Una encuesta de satisfacción permitirá conocer si lo impartido les aporta o no conocimientos, habilidades y actitudes en cuanto a la conexión, interacción y uso de la información; si experiencias como éstas serían útiles dentro del plan de estudio; y opiniones sobre cómo llevar la formación en este sentido.

El profesional de la información debe confeccionar materiales de apoyo para su inclusión en plataformas virtuales. Una vez accionado sobre los profesionales de la información, los docentes y los estudiantes, debe diseñarse un programa que integre la formación de competencias informacionales en las titulaciones dentro de asignaturas propias y/o en asignaturas específicas. Donde esta formación, llevada a cabo por los profesionales de la información, se conciba a lo largo de los cinco años de pregrado y sea gradual, aumentando el nivel de especialización. Y tenga en cuenta la valoración de los conocimientos previos de los destinatarios, los contenidos contextualizados a la situación de aprendizaje y la evaluación de los resultados.

\section{CONCLUSIONES}

La sociedad de la información y del conocimiento impone que los profesionales de la información sean competentes en información y aprendan a enseñar competencias informacionales. 
La gestión de competencias informacionales en la universidad conlleva a ampliar la dimensión educativa de la Biblioteca, colaborar en la formación de competencias y contribuir a que la formación de los egresados se corresponda con un mercado cada vez más exigente.

El profesional de la información deja de ser intermediario entre la información y el usuario y difusor de las fuentes de información; y se convierte en agente de la formación a lo largo de la vida para generar resultados en la sociedad.

Evidentemente se abre un camino apasionante, un reto para el profesional de la información; que requiere formación continua, abrirse a nuevas tareas y responsabilidades, y aliarse a otros profesionales de la información, a docentes y a estudiantes.

\section{REFERENCIAS}

ACRL. (2000). Information Literacy Competency Standards for Higher Education. [Citado el: 25 de enero de 2007.] http://www.ala.org/acrl/ilcomstan.html.

Agudelo, S. (1997). La certificación: duración, transferibilidad e instituciones. Seminario Formación basada en competencias. Situación actual y perspectivas para los países del MERCOSUR. [En línea] 1997. [Citado el: 20 de septiembre de 2006.] http://www.oei.es/oeivirt/fp/cuad2a05.htm.

Ameida de, V. (1991). Encino para competencia e auto-instrucao. Caracterizacao das duas metodologias. Boletím Técnico do SENAC. 1991, Vol. 17, 3, págs. 269-279.

Angulo, N. (2003). Normas de competencia en Información. BiD: textos universitaris de Biblioteconomia $i$ Documentació Núm.11. [Citado el: 15 de enero de 2007.] http://www2.ub.es/bid/consulta_articulos.php?fichero=11angul2.htm.

ANZIIL. (2004). El marco para la alfabetización informacional en Australia y Nueva Zelanda. Principios, normas y práctica. [Citado el: 24 de marzo de 2006.] http://www.aab.es/pdfs/baab73/73a4.pdf.

Badke, W. (2008). A rationale for information literacy as a credit-bearing discipline. Journal of information literacy 2(1). [Citado el: 14 de junio de 2009.] http://jil.lboro.ac.uk/ojs/index.php/JIL/article/view/RA-V2-I1-2008-1.

Beedle, P. (1997). La experiencia de la Unión Europea I. Seminario Formación basada en competencias. Situación actual y perspectivas para los países del MERCOSUR. [Citado el: 24 de junio de 2006.] http:/ / www.oei.es/oeivirt/fp/cuad2a05.htm.

Benito, F. (2000). Nuevas necesidades, nuevas habilidades. Fundamentos de la alfabetización en información. En Gómez, J. A. (coord.) Estrategias y modelos para 
enseñar a usar la información: guía para docentes, bibliotecarios y archiveros. [Citado el: 14 de octubre de 2006.] http:/ / eprints.rclis.org/archive/00004672/02/EMPEUIcap1.pdf.

Bruce, C. (1997). Seven Faces of Information Literacy in Higher Education. Queensland University of Technology. [Citado el: 13 de marzo de 2007.] http://sky.fit.qut.edu.au/\%7Ebruce/inflit/faces/faces1.htm.

Cardona, P. y Chinchilla, M. N. (1999). Evaluación y desarrollo de las competencias directivas. Harvard Deusto Business Review, núm. 89. [Citado el: 28 de septiembre de 2006.]

http:/ / kino.iteso.mx/ luisg/EVALUACION\%20Y\%20DESARROLLO\%20DE\%20LAS \%20COMPETENCIAS\%20DIRECTIVAS.doc.

Catalana, A., Avolio de Cols, S. y Sladogna, M. (2004). Diseño curricular basado en normas de competencia laboral. Boletín Cinterfor. [Citado el: 15 de mayo de 2006.] http:// www.cinterfor.org.uy/public/spanish/region/ampro/cinterfor/publ/dis_cur r/pdf/dis_curr.pdf.

CAUL. (2001). Information Literacy Standards. [Citado el: 25 de enero de 2007.] http://www.caul.edu.au.

Christiansen, L., Stombler, M. y Thaxton, L. (2004). A report on librarian-faculty relations from a sociological perspectiva. The journal of academic librarianship. 2004, Vol. $30,2$.

Doyle, C.S. (1992). Outcome measures for information literacy within the National Education Goals. Final report to the National forum on Information Literacy. [Citado el: 15 de enero de 2007.]

http:// www.eric.ed.gov/ERICDocs/data/ericdocs2sql/content_storage_01/0000019b /80/13/2c/6e.pdf.

Ducci, M. (1997). El enfoque de competencia laboral en la perspectiva internacional. Formación basada en competencia labora. Montevideo : Cinterfor/OIT, 1997.

Dudziak, E. A. (2003). Information literacy: principios, filosofía e practica. Ciencia da Informacao. 2003, Vol. 32, 1.

Edwards, S. 2006. Panning for Gold. Information Literacy and the Net Lenses Model. Adelaide : Auslib Press.

Eisenberg, M.B. y Berkowitz, R.E. (1990). Information Problem Solving: The Big Six Skills Approach to Library and Information Skills Instruction. Norwood : Ablex.

Espinós, M. y Sort, M. Informar y formar o las dos caras de la misma moneda: la formación de usuarios en la Biblioteca de la Universitat Pompeu Fabra. [Citado el: 15 de mayo de 2012.] http:www.upf.edu/bib/coneixer/publicac/informar.htm 
Frías, M. (2000). La formación de bibliotecarios universitarios en Cuba. La Habana: Universidad de La Habana, 2000. Trabajo de Diploma para obtener el título de Licenciado en B-CI.

Gallart, M. A. y Jacinto, C. (1995). Competencias laborales: tema clave en la articulación educación-trabajo. Boletín Educación y Trabajo año 6, núm. 2. [Citado el: 3 de marzo de 2006.] http://www.campus-oei.org/oeivirt/fp/cuad2a04.htm.

García, C., Irigoyen, R. M. y Llorens, A. (2008). Alfabetización informativa: ¿proyecto o quimera? Crítica Bibliotecológica. 2008, Vol. 1, 1.

Gómez-Hernández, J. A. (2008). Aprender a enseñar competencias informacionales a los usuarios: avancees en la formación profesional en España. Citado el 17 de noviembre de 2008. http://www.thinkepi.net/aprender-a-ensenar-competenciasinformacionales-a-los-usuarios-avances-en-la-formacion-profesional-en-espana.

Hager, P, Gonczi, A. y Athanasou, J. (1994). General Issues about assessment of competence. Assessment and Evaluation in Higher Education. 1994, Vol. 9, 1, págs. 3-16.

Hartzell, G.N. (1997). The invisible schoollibrarian: why other educators are blind to your value. School librar. School Library Journal. 1997, Vol. 43, 11.

Herring, J. (1996). Teaching information skills in schools. London: Library Association Publishing.

Ibarra, A. (2000). Formación de los Recursos Humanos y Competencia Laboral. Boletín Cinterfor, núm. 149. [Citado el: 28 de septiembre de 2006.]

http://www.cinterfor.org.uy/public/spanish/region/ampro/cinterfor/publ/boletin $\angle 149 /$ pdf/ibarra.pdf.

INEM. (1995). Metodología para la ordenación de la formación profesional ocupacional. Subdirección general de gestión de formación ocupacional. Madrid : s.n., 1995. Citado por: IRIGOIN, M.E. y VARGAS, F. 2002. Competencia Laboral: manual de conceptos, métodos y aplicaciones en el sector salud. CINTERFOR - OPS. [En línea] 2002. [Citado el: 13 de mayo de 2006.]

http://www.cinterfor.org.uy/public/spanish/region/ampro/ci.

Irigoin, M. y Vargas, F. (2002). Certificación de competencias. Del concepto a los sistemas. Boletín Cinterfor núm. 152. [Citado el: 24 de junio de 2006.] http://www.cinterfor.org.uy/public/spanish/region/ampro/cinterfor/publ/boletin $\angle 152 /$ pdf/ir_va.pdf.

Irigoin, M. E. (1998). Teoría y práctica de las competencias. Washington : s.n., 1998. Reunión de Trabajo sobre Competencias. 
Jornet, J., Perales, M. J. y Pérez, A. (2002). Evaluación, acreditación y certificación de la formación profesional, ocupacional y continua: unas notas para la reflexión. Revista de Treball, Economia i Societat núm.24. [Citado el: 25 de enero de 2007.]

Kuhlthau, C.C. (1989). Information Search Process: a summary of research and implications for School Library Media Programs. School Library Media Quarterly 22(1). [Citado el: 25 de enero de 2007.] http:/ / www.ala.org/ala/aasl/aaslpubsan.

Laur-Ernst, U. (2000). Flexibilidad y normalización no son contradictorias. Innovaciones en el Sistema Alemán de Educación y Formación Profesional. Boletín Cinterfor núm. 149. [Citado el: 13 de marzo de 2006.] http://www.cinterfor.org.uy/public/spanish/region/ampro/cinterfor/publ/boletin $\angle 149 /$ pdf/utelau.pdf.

Lupton, M. (2004). The Learning Connection. Information Literacy and the Student Experience. Adelaide : Auslib Press.

Markless, S. (2008). Information Literacy: refocussing on learning and student choice in the electronic environment. [Citado el: 13 de marzo de 2009.] http:/ / www.scribd.com/doc/21980664/IL-Refocusing-on-Learning-and-StudentChoice-Sharon-Markless.

Marland, M. (1981). Information Literacy Skills in the Secondary Curriculum: The Recommendations of a Working Group Sponsored by the British Library and The School Council. London : Metheum Educational, 1981. Citado por: Benito, F. (2000). Nuevas necesidades, nuevas habilidades. Fundamentos de la alfabetización en información. En: Gómez, J.A. (coord.) Estrategias y modelos para enseñar a usar la información: guía para docentes, bibliotecarios y archiveros..

Martínez, D. (2005). El nuevo concepto de biblioteca universitaria. Jornadas "La calidad en las Bibliotecas". [Citado el: 24 de marzo de 2006.] http:/ / www.ucm.es/BUCM/biblioteca/doc6161.pdf.

McDonald, R., y otros. (1995). Nuevas perspectivas sobre la evaluación. Sección para la Educación Técnica y Profesional. Boletín Cinterfor 2000, Núm. 149. [Citado el: 15 de enero de 2006.]

http://www.cinterfor.org.uy/public/spanish/region/ampro/cinterfor/publ/boletin $\angle 149 /$ pdf/rodajog.pdf.

Mertens, L. (1998). La gestión por competencia laboral y la formación profesional. Programa de Cooperación Iberoamericana para el diseño de la formación profesional. Cumbre Iberoamericana.

Mirabile, R. J. (1997). Everything you wanted to know about competency. Training and Development. 1997, págs. 73-77. 
Pappas, M. y Tepe, A. (1995). Preparing the information educator for the future. School Library Media Annual. 1995, 13. Citado por: Ortoll, E. (2003a). Competencia informacional en las ciencias de la salud. Propuesta de un modelo de formación. Universidad de Zaragoza. España : s.n., 2003a. Tesis doctoral.

Pasadas, C. y Álvarez, F.J. (2007). Formación de los ciudadanos en competencias para el manejo de la información: una propuesta de actuación para Andalucía. Resumen ejecutivo. Jornadas Bibliotecarias de Andalucía. [Citado el: 26 de noviembre de 2008.] http:// www.aab.es/pdfs/jba14/PasadasAlvarez.pdf.

Pinto, M., Doucet, A.V. y Fernández-Ramos, A. $\left(2008^{a}\right)$. The role of information competencies and skills in learning to abstract. Journal of Information Science. 2008a, Vol. 34, 6, págs. 799-815.

Pinto, M., Sales, D. y Martínez-Osorio, P. 2008b. Biblioteca universitaria, CRAI y alfabetización informacional. Gijón : Trea, 2008b.

-. $\left(2009^{\mathrm{a}}\right)$. El personal de la biblioteca universitaria y la alfabetización informacional: de la autopercepción a las realidades y retos formativos. Revista Española de Documentación Científica. 2009a, Vol. 32, 1, págs. 60-80.

Pinto, M., y otros. (2009b). Alfabetización múltiple desde la biblioteca pública: experiencias y propuestas. Buenos Aires : Alfagrama.

Roca, M. (2006). El bibliotecario temático y formador. Proyecto de la Universitat Politècnica de Catalunya. Educación y Biblioteca. 2006, Vol. 18, 156.

Sánchez, M. (2004). El profesional de la información en el ámbito iberoamericano. Acimed. 2004, Vol. 12, 2.

SCONUL. (1999). Information skills in higher education a SCONUL Position Paper. [Citado el: 15 de enero de 2007.] http:/ / www.sconul.ac.uk/.

Stripling, B.K. y Pitts, J.M. (1988). Brainstorms and Blueprints. Englewood: Libraries Unlimited. Citado por: Ortoll, E. (2003 $\left.{ }^{\mathrm{a}}\right)$. Competencia informacional en las ciencias de la salud. Propuesta de un modelo de formación. Universidad de Zaragoza. España : s.n., 2003a. Tesis doctoral .

Tejada, J. (1999). Acerca de las Competencias Profesionales. Revista Herramientas núm. 57. [Citado el: 29 de junio de 2007.] http://dewey.uab.es/pmarques/dioe/competencias.pdf.

Tucker, M. y Brown, B. (2000). Un sistema nacional de normas de competencia y certificaciones para los Estados Unidos: etapas iniciales de implementación. Boletín Cinterfor núm.149. [Citado el: 13 de marzo de 2007.] 
http://www.cinterfor.org.uy/public/spanish/region/ampro/cinterfor/publ/boletin $\angle 149 /$ pdf/tuc_bro.pdf.

Vargas, F. (2004). 40 preguntas sobre competencia laboral. [Citado el: 13 de marzo de 2006.]

http://www.cinterfor.org.uy/public/spanish/region/ampro/cinterfor/publ/papel/ 13/index.htm.

Vargas, F., Casanova, F. y Montanaro, L. (2001). El enfoque de competencia laboral: manual de formación. [Citado el: 12 de marzo de 2007.] http://www.cinterfor.org.uy/public/spanish/region/ampro/cinterfor/publ/man_cl Lindex.htm.

\section{Marlery Sánchez Díaz}

Es Doctora en Ciencias de la Documentación por la Universidad de Granada, en España. En la actualidad ejerce como Profesora Asistente Adjunta en el Departamento de Ciencias de la Información de la Facultad de Comunicación de la Universidad de La Habana, en Cuba. También es Especialista en Información Científico-Técnica y Bibliotecología y Tecnólogo de Avanzada I nivel en el Centro Nacional de Biopreparados (BioCen) en La Habana. 